\title{
Assessment of Serum L-Ficolin Level as Inflammatory Marker and Its Correlation with Serum C-Reactive Protein, D-Dimer and Ferritin in a Sample of Iraqi COVID-19 Patients
}

\author{
Authors \\ *Layth Taha Abdulhussein, **Dr. Mohammed Abdullatif, ***Dr. Aref Sami Malik \\ Collage of Medicine - Al-Nahrain University - Baghdad -Iraq \\ Contact us: E-mail / alimsaleam@gmail.com \\ Mobile / 07725503941
}

\begin{abstract}
Subject: At the end of December 2019, a COVID-19 outbreak was reported for the first time in Wuhan, China. Which quickly expanded throughout China, then to 209 nations across America, Europe, Australia, and Asia. More than 50,000 people have died and over one million individuals have been afflicted worldwide, with the number fast rising. COVID-19 has been combated in various ways around the world. The gold standard for COVID-19 diagnosis is real-time fluorescence quantitative reverse transcriptase polymerase chain reaction (reverse transcriptase-polymerase chain reaction, RT-PCR) and viral gene sequencing. Currently, nasopharyngeal swabs from the upper respiratory tract are the most commonly employed nucleic acid detection samples.

Objective of the Study: To estimate the level of ficolin in covid-19 patient in moderate and severe disease stage determination (series measurement and follow up) and find the diagnostic tool to early detect the virus to minimize the severity of the disease and further complication. Correlation of serum L-ficolin concentrations with Serum C-Reactive Protein, D-Dimer and Ferritin also to find Prognostic value of L-ficolin will be studied by corelating the treatment outcomes over one month with the three initial L-ficolin level.

Materials and Methods: Sixty-two patients will be involved in the study from both sex male and female and different age group (18-80 yr.), the basal level of ficolin will be measure at time of admission of patient (time zero), then further serial measurement will be done on day 7 of admission for precise ficolin monitoring.

Results: This study shows elevation of serum L-Ficolin level in survival and non-survival group.

Conclusion: The results showed significant negative correlation between L-ficolin levels with the duration of illness $(r=-0.377, p=0.020)$, however there were no significant correlation between L-ficolin levels with other patient's parameters.
\end{abstract}

Keywords: L-Ficolin, Corona Virus, SARS-CoV2, Complement, Lectin.

\section{Introduction}

Coronavirus Infection (Covid-19). A new form of coronavirus (SARSCoV-2) was discovered in 2020. This virus was identified for the first time in Wuhan, China, on March 2, 2020, and has infected 90,308 people. The number of people who died reached 3,087 , or $6 \%$ of the total number of patients who were rescued, which was 45,726 . This single positive RNA strain infects the human respiratory tract, is heat sensitive, and can be triggered effectively by chlorine-based disinfectants. (Glass, Cash and 
Mullen, 2020). According to recent reports, the magnitude of some Covid-19 cases resembled that of SARS-CoV. Given Covid-19's rapid spread. (Huang et al., 2020). The following is a timeline of COVID-19 infections, The first cases were reported in December 2019 (Antonio et al., 2003). From December 18, 2019 through December 29, 2019, five patients were hospitalized with acute respiratory distress syndrome and one of these patients died (Ren et al., 2020). By January 2, 2020, 41 admitted hospital patients had been identified as having laboratoryconfirmed COVID-19 infection cardiovascular disease. (Huang et al., 2020). After an incubation time of about 5.2 days, COVID-19 infection symptoms appear. (Li et al., 2020). The time it took for COVID-19 symptoms to manifest into death ranged from 6 to 41 days, with a median of 14 days, the length of time depends on the patient's age and the state of his or her immune system.

By comparing patients over the age of 70 to those under the age of 70 , it was shorter . (Wang, Tang and Wei, 2020). Fever, cough, and fatigue are the most common symptoms of COVID-19 infection, but other symptoms include sputum production, headache, hemoptysis, diarrhea, dyspnea, and lymphopenia.(Huang et al., 2020) (Wang, Tang and Wei, 2020) (Chang et al., 2020)

on the other hand, COVID-19 displayed several distinct clinical characteristics, such as the targeting of the lower airway, as shown by upper respiratory tract symptoms such as rhinorrhea, sneezing, and sore throat. (Antonio et al., 2003). Lymphopenia, leukopenia, thrombocytopenia, elevated CRP and inflammatory markers, elevated cardiac biomarkers, reduced albumin, and irregular renal and liver function are the most common laboratory anomalies in patients with positive RT-PCR. (Zhu et al., 2020). Furthermore, some of the cases display an infiltrate in the upper lobe of the lung, which is linked to increased dyspnea with hypoxemia based on the findings of chest radiographs taken upon admission. Importantly, although COVID-19 patients encountered gastric symptoms such as diarrhea, only a small percentage of MERS-CoV or SARS-CoV patients experienced similar GI distress. As a result, it's critical to screen feces and urine samples to rule out a possible alternative route of transmission, such as by health-care staff, patients, and so on. (Phan et al., 2020). The immune system is a set of cells and molecules that play specific roles in fighting infection. Invading microbes elicit two fundamentally different forms of responses. Innate (natural) responses are the same no matter how many times an infectious agent is encountered, while acquired (adaptive) responses strengthen with repeated infection exposure.(Peter J. Delves, 2000). The complement system is the first to react innately. When complement molecules in the plasma bind to specific bacteria, they aid in their 
elimination by lysis or opsonization. Lysis is the mechanism in which complement ruptures the bacterial membrane, causing the bacterium to die. Opsonization is the process of covering bacteria with complement (or antibodies; so that macrophages can detect them. On the surface of selfcells are regulatory proteins that prevent complement from binding to them. As a result, they are shielded from complement's effects. In the early stages of infection, complement and macrophages are usually activated within the first few hours. (Hofmeyr, 2000). Complement was first identified in the 1890s recognized as a highly sophisticated host defense system that is activated by innate immunity as well as one of the key effector mechanisms of antibody-mediated immunity.(Fujita, 2002). The complement system is involved in innate immunity and can be activated through three different pathways: classical, alternative, and lectin pathways. Complement activation through the lectin pathway is an important component of innate antimicrobial host protection.(Lynch et $a l .$, 2004). Mannan-binding lectin (MBL) or ficolins, which are patternrecognition molecules capable of recognizing conserved pathogenassociated molecular patterns (PAMPs) shared by a wide number of microorganisms, activate the lectin pathway. As a result, the hosts are effectively protected from infection. Binding of lectin pathway recognition molecules (MBL or ficolins) to microbial carbohydrates can activate complement through a mechanism similar to the classical pathway, but instead of $\mathrm{C} 1 \mathrm{r}$ and $\mathrm{C} 1 \mathrm{~s}, \mathrm{MBL}$ associated serine proteases are used. (Holmskov, Thiel and Jensenius, 2003). L-ficolin/P35 (FCN2 or ficolin 2). (Matsushita et al., 1996), M-ficolin (FCN1 or ficolin 1). (Endo et al., 1996), and $\mathrm{H}$-ficolin/Hakata antigen (FCN3 or ficolin 3). (Yae et $a l ., 1991)$ have recently been identified in humans. L-ficolin is produced mainly in the liver and then released into the bloodstream. (Zhang and Ali, 2008). The ligand specificities for ficolins and MBL, however, are different. MBL has a good reaction with mannose and fucose, while Lficolin binds to GlcNAc but not to mannose. L-ficolin binds to the GlcNAc residue next to galactose at the oligosaccharide's nonreducing terminal. (Ma et al., 2004). Following complement activation, L-ficolin was found to bind to a variety of acetylated compounds, including 1,3-D glucan, a molecular marker of yeast and fungal cell walls, lipopolysaccharide (LPS) on gram-negative bacteria, and Lipoteichoic Acid (LTA) on gram-positive bacteria Staphylococcus aureus and Streptococcus pneumoniae. (Krarup et al., 2004). 


\section{Materials and Methods}

The present study included 62 cases with severe and acute severe disease, all individuals age is between (18-80) years of both sexes. The sampling process of all subjects was random manner, blood samples separated immediately without any preservative factor to product serum as pure form. The serum used for measured Adiponectin concentration level (as quantity measurement )by immunoassay method. Also, the level of CRP, Ferritin, LDH and D-dimer was also measured

After measurement, independent sample t-test (unpaired t-test between two groups), paired sample t-test (between first and second measurements), chi square (for non-continuous data or percentage) and repeated measured ANOVA (evaluate interactions between patients groups and time of measurements). to show different of L-ficolin level by comparison between series measurement via used mean \pm standard deviation (SD) and $p$-value ( $p$-value $>0.05$ mean significant value ).The data were analyzed using Statistical Package for Social Sciences (SPSS) version 23.0 and Microsoft office 2007.Statistical data including frequency, mean and standard deviation were measured to describe the variables

\section{Results}

The present study explains a different of L-ficolin concentration level between COVID-19 patients for survival and non-survival group. The correlations between L-ficolin measurements with patient's age, duration of illness, D-dimer, LDH, ferritin and CRP in men were demonstrated in table 1 . The results showed significant negative correlation between Lficolin levels with the duration of illness $(r=-0.377, p=0.020)$, however there were no significant correlation between L-ficolin levels with other patient's parameters.

. Show table 1 .

Table 1: Correlation between L-ficolin levels with patient's parameter in male

\begin{tabular}{|c|c|c|}
\hline \multirow{2}{*}{ AGE } & $r$ & \multicolumn{1}{|c|}{ L-ficolin $1^{\text {st }}$ reading } \\
\hline & $p$ value & 0.239 \\
\cline { 2 - 4 } & $r$ & 0.160 \\
\hline Duration of illness & $p$ value & -0.377 \\
\cline { 2 - 4 } & $r$ & $0.020^{*}$ \\
\hline D-dimer (mg/l) & & -0.177 \\
\hline
\end{tabular}




\begin{tabular}{|c|c|c|}
\hline & $p$ value & 0.326 \\
\hline \multirow{2}{*}{ LDH (U/I) } & $r$ & -0.025 \\
\cline { 2 - 4 } & $p$ value & 0.900 \\
\hline Ferritin (ng/ml) & $r$ & 0.095 \\
\cline { 2 - 4 } & $p$ value & 0.603 \\
\hline CRP $(\mathrm{mg} / \mathrm{l})$ & $r$ & -0.082 \\
& & 0.631 \\
\cline { 2 - 4 } & $p$ value & \\
\hline
\end{tabular}

*Significant value

The comparison between male and female L-ficolin levels were presented in table 2 and figure 2. There was lower L-ficolin levels in women (139.97 versus 135.98) and (146.87 versus 116.37) respectively, however there was no significant difference in L-ficolin measurements between men and women $(\mathrm{p}>0.05)$.

Table 2: Comparison of L-ficolin levels between male and female

\begin{tabular}{|c|c|c|c|}
\hline Parameter & $\begin{array}{c}\text { Male } \\
(\text { Mean } \pm \text { SD })\end{array}$ & $\begin{array}{c}\text { Female } \\
(\text { Mean } \pm \text { SD })\end{array}$ & $p$ value \\
\hline $\begin{array}{c}\text { L-ficolin } 1^{\text {st }} \text { reading } \\
(\mathrm{ng} / \mathrm{ml})\end{array}$ & $139.97 \pm 92.75$ & $135.98 \pm 70.29$ & 0.839 \\
\hline $\begin{array}{c}\text { L-ficolin } 2^{\text {nd }} \\
(\mathrm{ng} / \mathrm{ml})\end{array}$ & $146.87 \pm 103.83$ & $116.37 \pm 60.03$ & 0.205 \\
\hline
\end{tabular}

There was significantly higher number of non-survivals patients with increased second measurement of L-ficolin (4 versus 11) $(\mathrm{p}=0.038)$ as presented in table 3 so according to this an increase in the second measurement of 1-ficolin had bad prognosis.

Table 3-: Numbers of patients with increased and decreased second measurement of L-ficolin

\begin{tabular}{|c|c|c|c|}
\hline Group & $\begin{array}{c}\text { Decreased levels } \\
\text { n. (\%) }\end{array}$ & $\begin{array}{c}\text { Increased levels } \\
\text { n. }(\%)\end{array}$ & \multirow{2}{*}{$0.038^{*}$} \\
\hline Survivals & $27(57.4 \%)$ & $20(42.6 \%)$ & \\
\hline Non-survivals & $4(26.7 \%)$ & $11(73.3 \%)$ & \\
\hline
\end{tabular}


Paired sample $t$ test and repeated measures ANOVA were applied to compare between first and second l-ficolin measurements and interaction with patients groups as presented in table 4, table 5 and figure 1 , accordingly there was no significant difference between the l-ficolin two measurements in survivals and non-survivals and no interaction between patients groups with time of 1- ficolin measurement, meaning there was no combined effect of measurement time and mortality together on 1ficolin levels.

Table 4: Paired sample t-test of L-ficolin measurements in survivals and non-survivals

\begin{tabular}{|c|c|c|c|}
\hline Group & $\begin{array}{c}\text { L-ficolin } 1^{\text {st }} \text { reading } \\
(\mathrm{ng} / \mathrm{ml})\end{array}$ & $\begin{array}{c}\text { L-ficolin } 2^{\text {nd }} \text { reading } \\
(\mathrm{ng} / \mathrm{ml})\end{array}$ & $p$ value \\
\hline Survivals & $143.9 \pm 88.1$ & $136.3 \pm 96.5$ & 0.470 \\
\hline Non-survivals & $114.7 \pm 67.9$ & $132.4 \pm 71.9$ & 0.154 \\
\hline
\end{tabular}

Table 5: Repeated measures ANOVA of L-ficolin measurements in survivals and nonsurvivals

\begin{tabular}{|c|c|c|c|}
\hline Group & $\begin{array}{l}\text { L-ficolin } 1^{\text {st }} \text { reading } \\
(\mathrm{ng} / \mathrm{ml})\end{array}$ & $\begin{array}{c}\text { L-ficolin } 2^{\text {nd }} \text { reading } \\
(\mathrm{ng} / \mathrm{ml})\end{array}$ & $p$ value \\
\hline Survivals & $143.9 \pm 88.1$ & $136.3 \pm 96.5$ & \\
\hline Non-survivals & $114.7 \pm 67.9$ & $132.4 \pm 71.9$ & \\
\hline$p$ value & \multicolumn{2}{|c|}{0.605} & \\
\hline
\end{tabular}

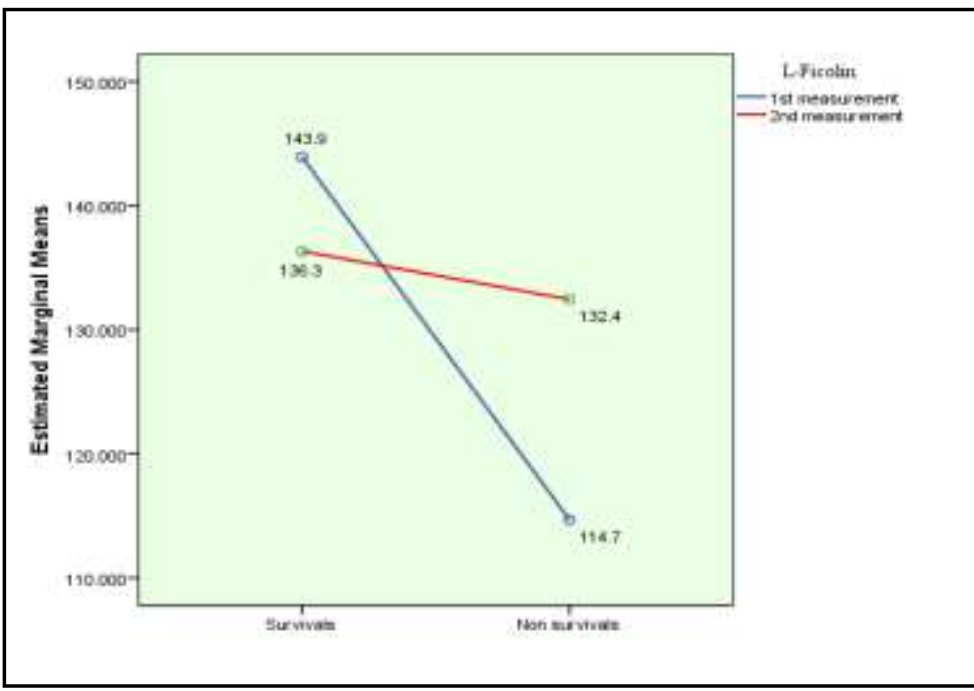

Figure 1: Comparisons of L-ficolin means in survivals and non-survivals 


\section{Discussion}

Accordingly, to this study, there is a weak positive correlation between age and L-ficolin level in male $(\mathrm{r}=0.239)$ with no significant difference $(p=0.160)$, mean while there is no correlation in L-ficolin level with serum Ferritin and CRP in male patient ( $\mathrm{r}=0.095), \quad(\mathrm{r}=-0.082)$ respectively. Interesting results shows negative correlation between duration of illness and L-ficolin level in male ( $\mathrm{r}=-0.377)$ with significance difference $(p=0.02)$, that is suggest low ficolin level gives shorter duration of illness and high survival rates.

The level of L-ficolin (first reading and second reading) in male was $(139.97 \pm 92.75),(146.87 \pm 103.83)$ respectively and the level of Lficolin (first reading and second reading) in female was (135.98 \pm 70.29$)$, $(116.37 \pm 60.03)$ respectively. There was lower L-ficolin levels in women (135.98 versus 139.97 ) and (116.37 versus 146.87), however there was no significant difference in L-ficolin measurements between men and women $(p>0.05)$.

In this study conduct in Dar Al-Salam hospital, we find the effect of second measurement of L-ficolin on survival rate. The increasing in second measurement of L-ficolin indicating bad prognosis and increase of mortality rate, there is significantly high number of non-survived patient with increased second measurement of L-ficolin. This is explain by the inflammatory response which plays a critical role in pathogenesis of COVID-19 patient and COVID-19 is made worse by an inflammatory cytokine storm. (Liu et al., 2020). Also approved that Cytokine storm is crucial to the progression of COVID-19 and can lead to severe complications and death. discovered that cytokine storm is critical for COVID-19 progression and can lead to serious complications and mortality.(Madewell et al., 2020).

L-ficolin is one of important protein of lectin complement pathway that is utilize during acute infection phase, to activate the complement system and considered the key contributor of innate immune system and inflammation which induced thrombosis which is the clinical feature of COVID-19. In this study we measure the L-ficolin (which is one of lectin pathway recognition molecule) level in series measurement ( at day of admission and in day 7 ), because of lectin protein do not differ significantly between younger and older patient, so we do not consider the age is a limitation factor (Lafon et al., 2020). The mean and standard deviation of L-ficolin level on admission day for survival patient and non-survival was $(145.8 \pm 88.05),(114.7 \pm 67.9)$ respectively and the mean and standard deviation of L-ficolin level on day 7 after admission for survival patient and non-survival was $(136.3 \pm 96.5),(132.4 \pm 71.9)$ respectively. The difference between two groups (survival and nonsurvival) for the first reading was not significantly high $(p=0.216)$, the 
difference between these two group for the second reading of L-ficolin level was not significantly high also $(P=0.887)$. these results suggest that the L-ficolin level reduced in survived group when we do the series measurement for severe or severe critical patient from first reading and second reading $(143.9 \pm 88.1,136.3 \pm 96.5)$ respectively. And the level of L-ficolin in non-survived group between first and second reading was increased $(114.7 \pm 67.9,132.4 \pm 71.9)$. Suggesting that the increase of Lficolin upon time predicted bad prognosis and increase the death rate mean while the reduction in L-ficolin level upon the time during acute infection state predict good indicator and increase survival rate.

These finding also agreed with study published, In the case of Ficolin 2 (L-ficolin), non-survivors had significantly greater baseline plasma levels than survivors upon admission to the ICU. MBL levels were significantly greater in survivors during the five days, progressively increasing in both groups - but more notably in the surviving group - when examining levels of PRMs in non-survivors and survivors upon admission (day 1) and on the next four days in the ICU. From day one to day three, survivors had significantly greater levels of ficolin-2.(Hansen et al., 2020).

\section{References}

Antonio, G. E. et al. (2003) 'Imaging of severe acute respiratory syndrome in Hong Kong', American Journal of Roentgenology, 181(1), pp. 11-17. doi: 10.2214/ajr.181.1.1810011.

Chang, D. et al. (2020) 'Time kinetics of viral clearance and resolution of symptoms in novel Coronavirus infection', American Journal of Respiratory and Critical Care Medicine, 201(9), pp. 1150-1152. doi: 10.1164/RCCM.202003-0524LE.

Endo, Y. et al. (1996) 'Cloning and characterization of the human lectin P35 gene and its related gene', Genomics, 36(3), pp. 515-521. doi: 10.1006/geno.1996.0497.

Fujita, T. (2002) 'Evolution of the lectin - Complement pathway and its role in innate immunity', Nature Reviews Immunology, 2(5), pp. 346353. doi: $10.1038 / \mathrm{nri} 800$.

Glass, C. A., Cash, J. C. and Mullen, J. (2020) 'Coronavirus Disease (COVID-19)', Family Practice Guidelines, (October). doi: 10.1891/9780826153425.0016b.

Hansen, C. B. et al. (2020) 'Complement related pattern recognition molecules as markers of short-term mortality in intensive care patients', Journal of Infection, 80(4), pp. 378-387. doi: 10.1016/j.jinf.2020.01.010. 
Hofmeyr, S. A. (2000) 'An interpretative introduction to the immune system', Design Principles for the Immune System and Other Distributed Autonomous Systems, 3, pp. 3-26. Available at: http://scholar.google.com/scholar?hl=en\&btnG=Search\&q=intitle:An+Int erpretative+Introduction+to+the+Immune+System\#0.

Holmskov, U., Thiel, S. and Jensenius, J. C. (2003) 'Collectins and ficolins: Humoral lectins of the innate immune defense', Annual Review of Immunology, 21, pp. 547-578. doi: 10.1146/annurev.immunol.21.120601.140954.

Huang, C. et al. (2020) 'Clinical features of patients infected with 2019 novel coronavirus in Wuhan, China', The Lancet, 395(10223), pp. 497506. doi: 10.1016/S0140-6736(20)30183-5.

Krarup, A. et al. (2004) 'L-ficolin is a pattern recognition molecule specific for acetyl groups', Journal of Biological Chemistry. doi: 10.1074/jbc.M407161200.

Lafon, D. C. et al. (2020) 'Classical and lectin complement pathways and markers of inflammation for investigation of susceptibility to infections among healthy older adults', Immunity and Ageing, 17(1), pp. 1-9. doi: 10.1186/s12979-020-00189-7.

Li, Q. et al. (2020) 'Early Transmission Dynamics in Wuhan, China, of Novel Coronavirus-Infected Pneumonia', New England Journal of Medicine, 382(13), pp. 1199-1207. doi: 10.1056/nejmoa2001316.

Liu, F. et al. (2020) 'Prognostic value of interleukin-6, C-reactive protein, and procalcitonin in patients with COVID-19', Journal of Clinical Virology, 127(April), p. 104370. doi: 10.1016/j.jcv.2020.104370.

Lynch, N. J. et al. (2004) 'L-Ficolin Specifically Binds to Lipoteichoic Acid, a Cell Wall Constituent of Gram-Positive Bacteria, and Activates the Lectin Pathway of Complement', The Journal of Immunology, 172(2), pp. 1198-1202. doi: 10.4049/jimmunol.172.2.1198.

Ma, Y. G. et al. (2004) 'Human mannose-binding lectin and L-ficolin function as specific pattern recognition proteins in the lectin activation pathway of complement', Journal of Biological Chemistry, 279(24), pp. 25307-25312. doi: 10.1074/jbc.M400701200.

Madewell, Z. J. et al. (2020) 'NOTE: This preprint reports new research that has not been certified by peer review and should not be used to guide clinical practice. 1', medRxiv, (165), pp. 1-13.

Matsushita, M. et al. (1996) 'A novel human serum lectin with collagenand fibrinogen-like domains that functions as an opsonin', Journal of Biological Chemistry, 271(5), pp. 2448-2454. doi: 10.1074/jbc.271.5.2448.

Peter J. Delves (2000) 'First of Two Parts THREE LEVELS OF DEFENSE', pp. 37-49. 
Phan, L. T. et al. (2020) 'Importation and Human-to-Human Transmission of a Novel Coronavirus in Vietnam', New England Journal of Medicine, 382(9), pp. 872-874. doi: 10.1056/nejmc2001272.

Ren, L. L. et al. (2020) 'Identification of a novel coronavirus causing severe pneumonia in human: a descriptive study', Chinese medical journal, 133(9), pp. 1015-1024. doi: 10.1097/CM9.0000000000000722.

Wang, W., Tang, J. and Wei, F. (2020) 'Updated understanding of the outbreak of 2019 novel coronavirus (2019-nCoV) in Wuhan, China', Journal of Medical Virology, 92(4), pp. 441-447. doi: 10.1002/jmv.25689.

Yae, Y. et al. (1991) 'Isolation and characterization of a thermolabile $\beta-2$ macroglycoprotein ("thermolabile substance" or 'Hakata antigen') detected by precipitating (auto) antibody in sera of patients with systemic lupus erythematosus', Biochimica et Biophysica Acta (BBA)/Protein Structure and Molecular, 1078(3), pp. 369-376. doi: 10.1016/01674838(91)90158-V.

Zhang, X. L. and Ali, M. A. M. (2008) 'Ficolins: Structure, function and associated diseases', Advances in Experimental Medicine and Biology, 632, pp. 105-115. doi: 10.1007/978-0-387-78952-1_9.

Zhu, J. et al. (2020) 'Clinicopathological characteristics of 8697 patients with COVID-19 in China: A meta-analysis', Family Medicine and Community Health, 8(2), pp. 1-11. doi: 10.1136/fmch-2020-000406. 